\title{
PÉNDULO DE PRUEBA PARA EL ESTUDIO DINÁMICO DE MODELOS ESTRUCTURALES
}

\author{
Jaime De-la-Colina ${ }^{(\mathbf{1})}$ y Jesús Valdés ${ }^{(\mathbf{1})}$
}

\begin{abstract}
RESUMEN
Se presenta y analiza un aparato sencillo para el estudio dinámico de modelos estructurales en laboratorio. El dispositivo consiste de un péndulo de periodo largo (aprox. $5.0 \mathrm{~s}$ ) formado por una plataforma que cuelga de un techo alto en la cual se coloca un generador de vibración forzada. Cuando un modelo se coloca sobre la plataforma, el movimiento de ésta simula un tipo particular de movimiento sísmico. Este sistema se utilizó para el estudio dinámico de modelos de acero representativos de estructuras torsionalmente desbalanceadas. Las respuestas medidas, tanto de la plataforma como de los modelos se analizan para entender y conocer la capacidad del péndulo de pruebas para simular movimientos sísmicos del terreno de características particulares. Los resultados indican que el péndulo de prueba genera excitaciones propias de movimientos sísmicos de banda angosta. Este aparato de bajo costo puede utilizarse para pruebas dinámicas de modelos representativos de sistemas estructurales y mecánicos.
\end{abstract}

Palabras clave: pruebas dinámicas, experimentos de laboratorio, dispositivo de pruebas

\begin{abstract}
A simple apparatus for the dynamic study of laboratory structural models is presented. It consists of a long period $(5.0 \mathrm{~s})$ pendulum formed by a hanging platform with a forced vibration generator. The platform movement simulates a particular seismic ground motion that excites the structural model attached to the pendulum platform. This apparatus was used for the dynamic study of steel structural models of torsion desbalanced structures. The measured responses, both of the platform and of the models, are analyzed in order to understand and to know the pendulum capacities for simulating particular seismic ground motions. The results showed that the pendulum generates excitations that resemble those of narrow band seismic ground motions. This low cost apparatus can be used for dynamic testing of structural and mechanical systems.
\end{abstract}

Keywords: dynamic test, laboratory experiments, tests device

\footnotetext{
Artículo recibido el 18 de mayo de 2009 y aceptado para su publicación el 15 de enero de 2010. Se aceptarán comentarios y/o discusiones hasta cinco meses después de su publicación

${ }^{1}$ Profesores. Facultad de Ingeniería. Universidad Autónoma del Estado de México. Toluca, México. E-mail: jcolina@uaemex.mx , jvaldes@uaemex.mx
} 


\section{INTRODUCCIÓN}

Las pruebas de campo y laboratorio son importantes para soportar o rechazar resultados teóricos (analíticos o numéricos). Más aún, las pruebas de laboratorio ayudan a los científicos a entender el comportamiento de sistemas estructurales y mecánicos, particularmente bajo comportamiento no lineal donde las soluciones analíticas pueden ser limitadas. Por ello, para entender el comportamiento no lineal de las estructuras durante sismos fuertes, los ingenieros usualmente confían en los resultados de pruebas dinámicas de modelos estructurales.

En cuanto al equipo de laboratorio utilizado en este tipo de pruebas, existen distintos dispositivos (Hudson 1970). Actualmente, el dispositivo preferido para probar estructuras sujetas a acciones sísmicas es la mesa vibradora. Este dispositivo consiste de una plataforma que se mueve debido a la acción de uno o varios actuadores (gatos hidráulicos) para simular, principalmente, movimientos sísmicos del terreno, registrados previamente. Las mesas vibradoras se pueden construir de distintos tamaños y capacidades así como con uno o más grados de libertad. La mayoría de estos dispositivos sólo permiten llevar a cabo pruebas con modelos a escala, sin embargo, existen algunas que debido a su tamaño y capacidad permiten probar estructuras de pequeñas dimensiones a escala real. Este tipo de aparatos deberían ser de uso común en universidades y centros de investigación en ingeniería sísmica, sin embargo, son caros, tanto en su costo de adquisición como en su operación y mantenimiento. De ahí la necesidad de desarrollar dispositivos más sencillos que permitan llevar a cabo pruebas dinámicas.

Las pruebas pseudodinámicas se basan en una técnica reciente para simular en laboratorio patrones de carga generales, incluyendo aquellos correspondientes a movimientos sísmicos del terreno (Takanashi et al. 1980; Mahin y Shing 1985). Este procedimiento de prueba incorpora mediciones (en línea) de la respuesta instante a instante que permiten resolver mediante una computadora las ecuaciones dinámicas del movimiento. De esta forma, el sistema aplica fuerzas al modelo de prueba mediante gatos hidráulicos. Éste es un dispositivo de aplicación general de cargas que puede utilizarse en casi cualquier sistema dinámico que se quiera estudiar (aviones, autos, estructuras, etc.). Debido a que las fuerzas se aplican lentamente, tanto la inercia como el amortiguamiento se incorporan indirectamente en las ecuaciones del movimiento. Este procedimiento de prueba también es costoso.

Existen algunos otros dispositivos que pueden usarse para estudiar las propiedades dinámicas de sistemas dinámicos. Entre éstos están los generadores de vibración forzada (excitadores), los cuales generalmente funcionan mediante la acción de dos masas rotacionales excéntricas que sirven para inducir fuerzas armónicas al sistema en el cual están colocados (detalles adicionales de estos dispositivos se presentan en la siguiente sección de este artículo).

La literatura (Hudson 1970; Yu et al. 2008) también reporta el uso de excitadores lineales (electromagnéticos o hidráulicos). Estos dispositivos están diseñados principalmente para generar fuerzas a frecuencias mayores que las que se registran durante la ocurrencia de temblores, además de que las intensidades de sus fuerzas resultantes son limitadas, por lo que no son de mucha utilidad en el estudio sistemas estructurales sometidos a acciones sísmicas. Otras formas sencillas de estudiar las propiedades dinámicas de las estructuras son: 1) pruebas de vibración libre, 2) vibración inducida por la carga viva y 3) vibración ambiental, principalmente.

Este artículo presenta y analiza un dispositivo sencillo y de bajo costo, desarrollado para realizar en laboratorio pruebas dinámicas simples de modelos de estructuras y componentes estructurales sometidos a movimientos similares a los registrados en suelos blandos (movimientos sísmicos de banda angosta). El aparato desarrollado consiste de un péndulo de periodo largo (aprox. $5.0 \mathrm{~s}$ ) formado por una plataforma 
colgada del techo del laboratorio en la cual se tiene colocado un generador de vibraciones forzadas. Cuando los modelos de prueba se colocan sobre la plataforma, ésta se mueve como una cimentación sometida a un movimiento particular del terreno. En este artículo se describe el aparato, incluyendo los registros de aceleraciones medidos en la plataforma. El péndulo de prueba se utilizó para probar modelos de acero representativos de estructuras torsionalmente desbalanceadas. Debido a que el dispositivo fue desarrollado para estudiar la respuesta y comportamiento de modelos estructurales ante solicitaciones sísmicas, también se presentan algunas de las respuestas de los modelos probados para mostrar el tipo de movimiento inducido por el péndulo. Se analizan, tanto la respuesta de la plataforma como la del modelo para conocer la factibilidad del empleo del dispositivo en el estudio de modelos estructurales sometidos a sismos.

En cuanto a la organización del artículo, inicialmente se presenta una descripción del péndulo de prueba. Posteriormente, se describen los modelos estructurales de acero probados. Los resultados se presentan en dos partes. La primera parte describe el movimiento de la plataforma del péndulo con el propósito de caracterizar el movimiento del suelo simulado. Los resultados de los modelos probados se presentan en la segunda parte. Al final se presentan las conclusiones del trabajo.

\section{PÉNDULO DE PRUEBA}

En esta sección se describe el dispositivo de prueba completo, el cual está formado por el péndulo y el excitador. Como se observa en la Figura 1, el péndulo de prueba consiste de una plataforma de acero, la cual cuelga del techo del laboratorio mediante cuatro tirantes de acero. Un excitador de vibración forzada con eje vertical de rotación está unido a la plataforma. La fuerza resultante del excitador actúa en dirección normal al plano de la Figura 1. Los modelos a ser ensayados se colocan sobre la plataforma del péndulo como se muestra en la misma figura. En este caso, los modelos de prueba se conectaron en forma articulada a la plataforma para evitar una interacción a flexión de sus columnas con la plataforma. Del mismo modo, para evitar que la torsión de los modelos se transmitiera a la plataforma durante las pruebas, se utilizaron dos guías metálicas que obligaron a que la plataforma sólo tuviese movimiento de traslación. Es interesante observar que la rotación de la plataforma, la cual se produce debido a la rotación del modelo, podría controlarse colocándole a la plataforma algún dispositivo que simule la rigidez rotacional y el amortiguamiento del suelo. En los experimentos que se reportan en este artículo no se permitió el giro de la plataforma, ya que el modelo analítico de referencia únicamente consideró movimiento traslacional del suelo en una dirección. No fue posible determinar la efectividad de las guías debido a que no se registró el giro de la plataforma, sólo su traslación. La fricción inducida por ellas se consideró como parte del amortiguamiento (viscoso) del sistema.

La Figura 2 muestra una imagen del péndulo de prueba en el laboratorio con uno de los modelos ensayados. La grúa se utiliza para evitar que el modelo se caiga en caso de que éste falle. Aunque se consideraron algunos otros esquemas para el dispositivo de prueba (por ejemplo, una plataforma montada sobre rieles), el péndulo fue seleccionado por los autores ya que resultó ser el más simple, confiable y económico de todos ellos.

El generador de fuerza es un excitador mecánico que induce una fuerza armónica en una dirección, en este caso a lo largo de la dirección de oscilación del péndulo. En la Figura 3 se muestra una imagen del excitador utilizado en estos experimentos. Este excitador es accionado mediante un motor eléctrico de corriente alterna cuya velocidad es regulada por un controlador electrónico. Además, la velocidad con que giran las masas es modificada por la relación en los diámetros de los engranes que conectan el motor con las masas giratorias. Más información del excitador se presenta en el trabajo de Acuña (2003). Existen 
otros tipos de excitadores que pueden usarse para probar estructuras (e.g., Yu et al. 2008; Nieto et al. 1970), pero el utilizado en este caso es compacto y se acomoda bien en la plataforma de prueba.

El desarrollado por Nieto et al. (1970) es un vibrador mecánico capaz de excitar independientemente los modos de vibración vertical, horizontal, de cabeceo y de torsión de la base en que se coloque. En teoría, las frecuencias de excitación de dicho aparato pueden ir desde 1 hasta 33 cps, y es posible aplicar una fuerza armónica de $5 \mathrm{t}(49.03 \mathrm{kN})$ o bien un par armónico máximo de $2.8 \mathrm{t}$-m (27.45 $\mathrm{kN}-\mathrm{m})$. Este excitador es más grande y permite más formas de excitación que el utilizado en el péndulo de prueba que aquí se describe.

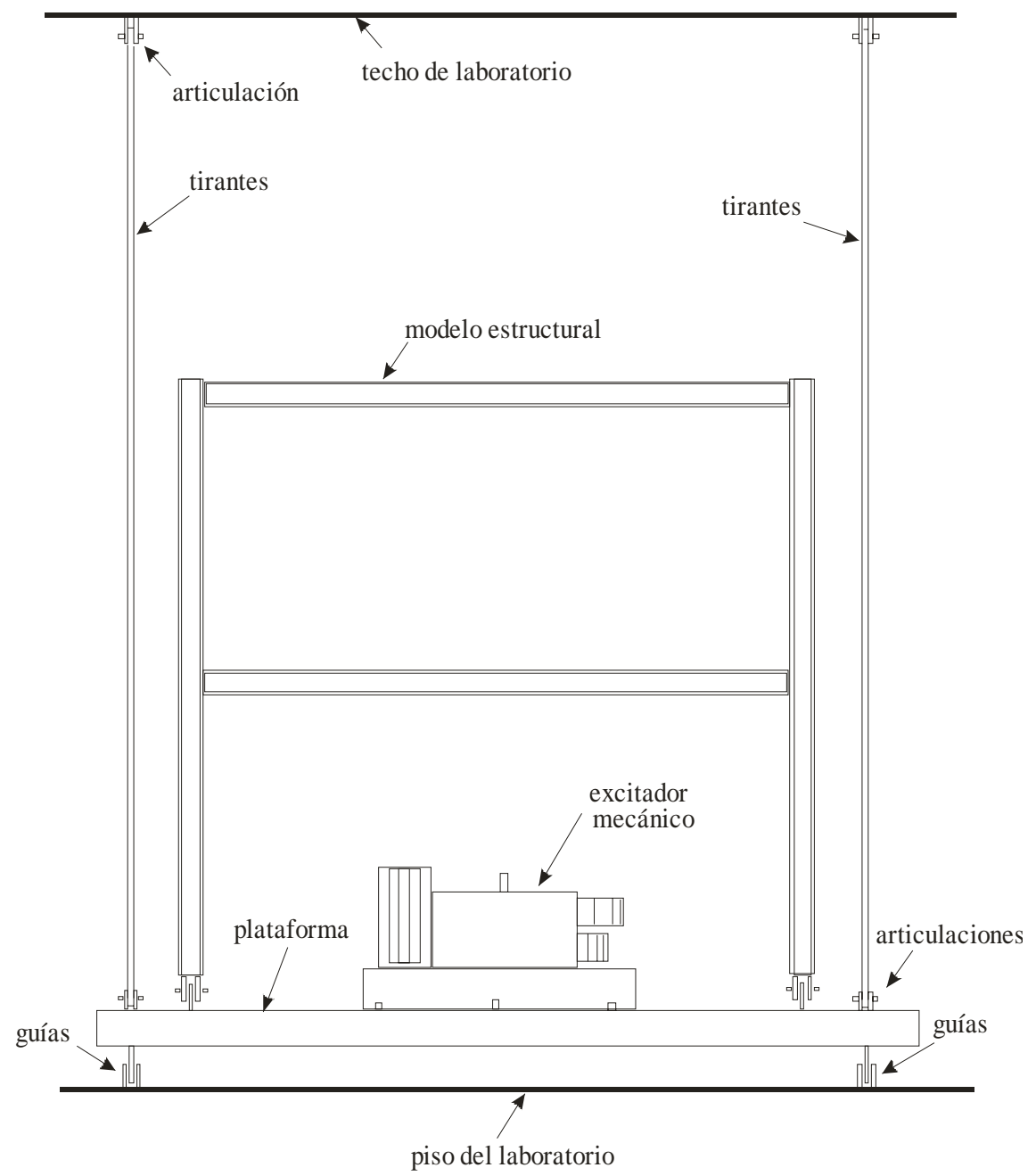

Figura 1. Péndulo de prueba con un modelo estructural.

Las mediciones obtenidas en el péndulo de prueba que se presenta en este trabajo indicaron que el periodo fundamental de vibración del sistema (péndulo, excitador y modelo) fue cercano a los $5 \mathrm{~s}$, el cual es consistente con los cálculos del periodo hechos con base en la longitud efectiva del péndulo. Esta longitud fue seleccionada para obtener un periodo de vibración del sistema significativamente mayor a los periodos de vibración de los modelos probados. Para el conjunto de pruebas inicialmente consideradas y reportadas en este artículo, los periodos de vibración de los modelos probados estuvieron cercanos a los 
0.6 s (ver Tabla 1 en la siguiente sección). Se puede considerar que la vibración de la plataforma y las de los modelos, prácticamente estuvieron desacopladas.

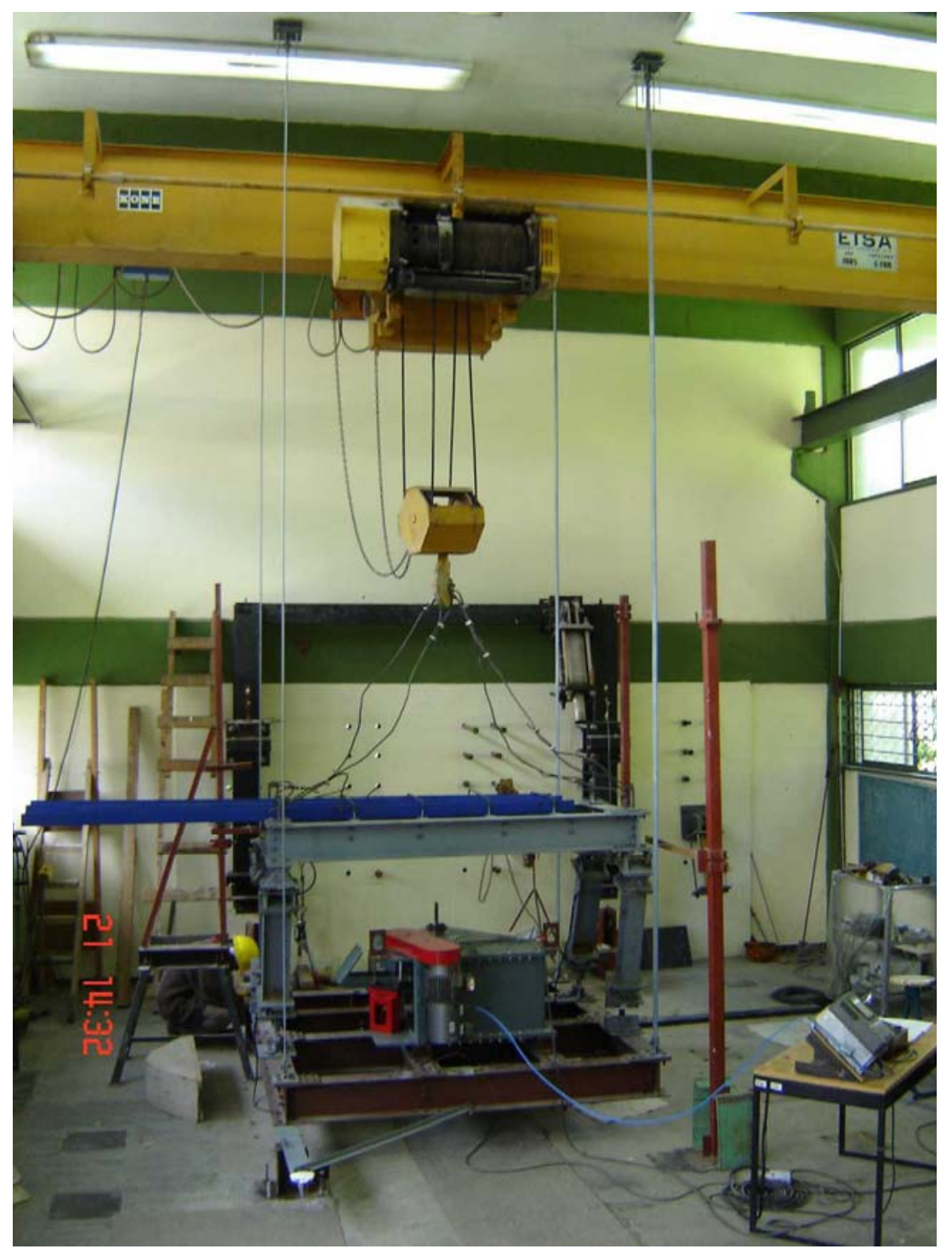

Figura 2. Péndulo de prueba con un modelo típico representativo de una estructura de un nivel.

La Figura 4 muestra una configuración típica de la plataforma del péndulo de prueba con un modelo estructural de un nivel sobre ella. Este sistema fue probado y las respuestas de la plataforma y del modelo en su parte alta fueron monitoreadas con acelerómetros, los cuales registraron la respuesta en la dirección de la excitación de la siguiente forma (ver Figura 4b): el acelerómetro 1 se colocó en la parte alta del modelo en el lado oeste, el acelerómetro 2 en la parte alta del modelo en el lado este y el 3 en la plataforma.

Las capacidades del péndulo de prueba fueron definidas para cubrir no sólo las necesidades de estas pruebas, sino que contemplan la posibilidad de ensayar otros modelos. Las capacidades actuales son las siguientes. Peso máximo del modelo a ensayar $=15 \mathrm{t}$. El intervalo de frecuencias de la excitación, el cual depende del excitador, es de $0.0 \mathrm{a} 10 \mathrm{~Hz}$. El desplazamiento lateral máximo de la plataforma es de $50 \mathrm{~cm}$, 
el cual conduce a un desplazamiento vertical de $6.0 \mathrm{~cm}$. La velocidad y la aceleración máximas de la plataforma son aproximadamente de $80 \mathrm{~cm} / \mathrm{s}$ y $100 \mathrm{~cm} / \mathrm{s}^{2}$, respectivamente.

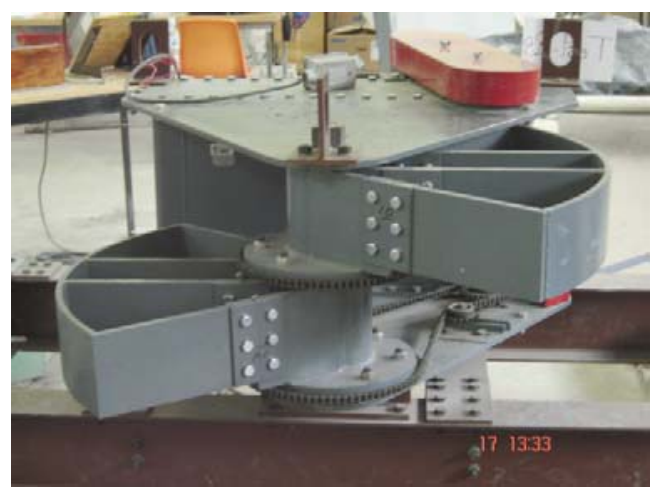

Figura 3. Generador de vibración forzada (excitador) colocado sobre la plataforma.

Antes de realizar las pruebas bajo vibración forzada, se realizaron algunas pruebas de vibración libre a los modelos, sin permitir movimiento de la plataforma. Estas pruebas sirvieron para determinar el amortiguamiento y las frecuencias naturales de vibrar $\omega_{i}$ de cada modelo. El porcentaje de amortiguamiento crítico $\left(\xi_{i}\right)$ estimado resultó cercano al $10 \%$ para todos los casos. Este amortiguamiento se estimó utilizando el método de decaimiento de la respuesta en vibración libre (Clough y Penzien 1993). Aunque este nivel de amortiguamiento parece alto para estructuras comunes de acero, cabe aclarar que los modelos ensayados tenían varias conexiones articuladas en los extremos de sus columnas que disiparon mayor energía en comparación a los casos típicos de conexiones estructurales. Newmark y Hall (1982) recomendaron valores de $\xi$ entre el $10 \%$ y el $15 \%$ para edificios de acero bajo comportamiento inelástico. De cualquier forma, el desempeño del péndulo de prueba no se afecta por este nivel de amortiguamiento.

Cada modelo (los modelos se describen en la siguiente sección) fue probado para tres diferentes relaciones de frecuencias $\Omega / \omega_{i}: 0.8,1.0$, y 1.2 , donde $\Omega$ es la frecuencia de la excitación que el generador impuso al sistema, como se comenta más adelante.

Durante cada prueba correspondiente a una relación de frecuencias en particular, la magnitud de la fuerza causada por el excitador se incrementó gradualmente agregando masas rotatorias a las canastas del excitador.

La fuerza que induce este tipo de generador puede expresarse como

$$
P(t)=p_{o} \operatorname{sen}(\Omega t)=\frac{W r}{g} \Omega^{2} \operatorname{sen}(\Omega t)
$$

donde $p_{o}=2 p$ es la amplitud de la fuerza, $W$ es el peso total de las masas rotacionales del excitador colocadas a una distancia $r$ del eje de rotación (ver el esquema simplificado del excitador en la Figura 5), y $g$ es la aceleración de la gravedad. Por ejemplo, para un modelo en particular (M6) que se probó a una frecuencia $\Omega=\omega_{6}=9.38 \mathrm{rad} / \mathrm{s}$ (Tabla 1) y $W r=43.45 \mathrm{~N}-\mathrm{m}(442.9 \mathrm{~kg}-\mathrm{cm})$, la amplitud de la fuerza resulta $p_{o}=390 \mathrm{~N}(39.8 \mathrm{~kg})$.

Agregando masas rotatorias al excitador, con lo cual se incrementó la magnitud de la fuerza aplicada, fue posible llevar los modelos a una etapa de comportamiento inelástico. Este tipo de 
comportamiento fue detectado midiendo los desplazamientos residuales del modelo después de las pruebas para cada incremento de masa rotatoria.

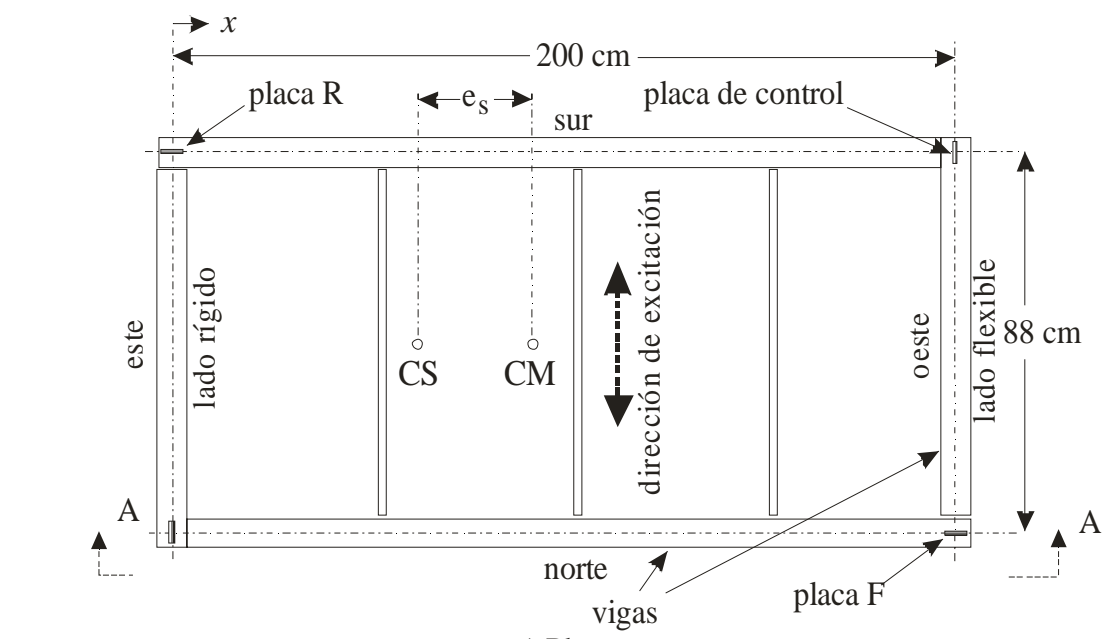

a) Planta

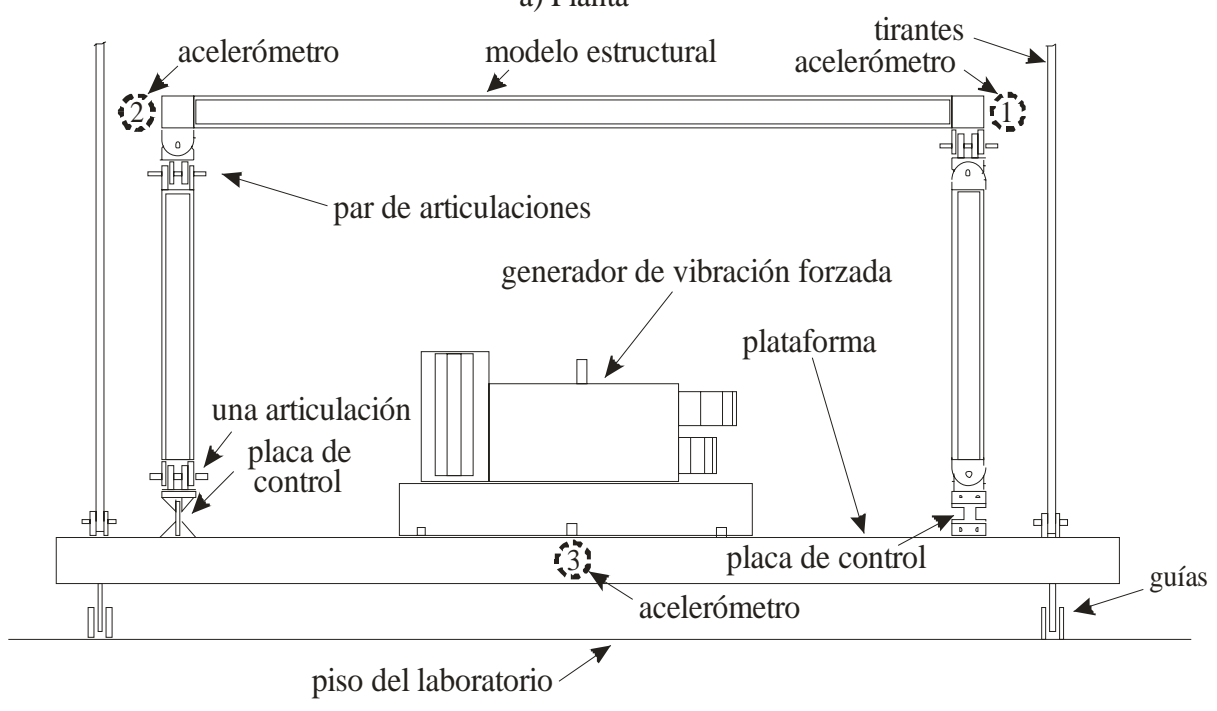

b) Vista lateral A-A

Figura 4. Plataforma del péndulo con un modelo de acero sobre ella.

\section{MODELOS PROBADOS}

Los modelos fueron probados para alcanzar dos objetivos. El primero fue estudiar el desempeño del péndulo de prueba. En particular, era importante identificar el movimiento que se presentaba en la base de los modelos para entender y conocer la capacidad del aparato para simular un movimiento del suelo en particular. El segundo objetivo fue estudiar la respuesta de modelos simples de edificios torsionalmente desbalanceados. Este artículo se enfoca a la descripción del péndulo de prueba, incluyendo su respuesta y comportamiento durante las pruebas. También se presenta la descripción general de la respuesta de los modelos, pero sólo para entender el tipo de movimiento generado por el aparato. Mayores detalles de la respuesta estructural de los modelos se pueden consultar en De-la-Colina et al. (2007). 

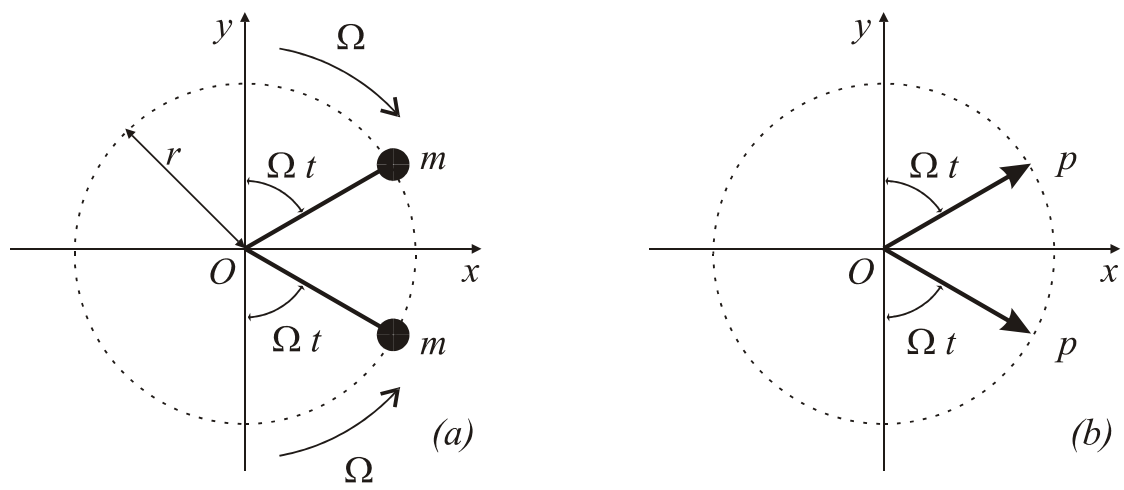

Figura 5. Principio de la generación de vibración forzada. Dos masas rotando a una misma velocidad alrededor de un eje común en sentidos opuestos.

Cada modelo consistió de una estructura de marcos de acero de un nivel con cuatro columnas y una cubierta formada por vigas I soportadas por las columnas (ver Figura 4). Estas vigas se conectaron a vigas secundarias para incrementar la rigidez en el plano de la cubierta. Cada columna tenía dos articulaciones perpendiculares en su extremo superior y sólo una articulación en su extremo inferior. El arreglo de las articulaciones en los extremos inferiores de las columnas fue tal que cada marco plano tenía dos columnas: una columna doblemente articulada sin resistencia lateral y una columna resistente en voladizo. En otras palabras, cada columna proveía resistencia lateral a lo largo de la dirección principal de la columna, mientras que en la dirección ortogonal no tenía resistencia lateral. De esta forma, la resistencia lateral de cada marco plano fue provista por una columna solamente. Se desarrollaron ocho modelos diferentes variando el ancho y el espesor de las placas (placas de control) que suministraban la única resistencia lateral a las columnas. Ver la localización de estas placas en la Figura 4b.

Se colocaron placas de control reemplazables de acero A-36 (con ancho $b_{p l}$ y espesor $t_{p l}$ ) en los extremos inferiores de las columnas, las cuales suministraron la resistencia lateral al modelo. Mediante una adecuada selección de las propiedades geométricas de estas placas se definieron las propiedades de resistencia y rigidez de cada columna. Este arreglo también permitió posicionar el centro de rigidez (CR) de cada modelo. En la Figura 6 se muestra una de estas placas de control después de haber fallado. Para verificar los valores de rigidez lateral se realizaron pruebas estáticas adicionales a cada columna.

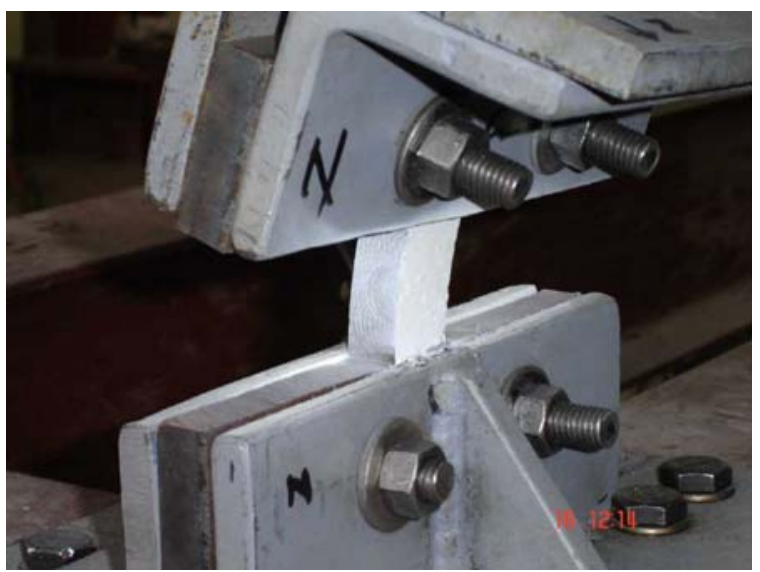

Figura 6. Placa de control en estado de falla. 
La dirección de excitación de los modelos fue paralela a sus lados cortos, como se indica en la Figura 4a. Por lo tanto, sólo las placas asociadas a esta dirección (marcos del lado rígido y flexible) fueron críticas. Estas placas de control se identifican como R (lado rígido) y $\mathrm{F}$ (lado flexible) en la misma figura. Las placas que suministraron resistencia a lo largo de la dirección ortogonal se propusieron con valores, que en promedio, resultaron similares a las placas R y F. De acuerdo a la recomendación de Humar y Kumar (1999), esta selección implicaría una relación de rigidez torsional $\gamma$ cercana a 0.89 para la geometría en planta seleccionada para los modelos. Esta relación se define como el cociente de la rigidez torsional de los planos paralelos a la dirección de la excitación entre la rigidez torsional total.

Se colocaron dos vigas de acero de sección canal (ver Figura 2) sobre la cubierta del modelo, de tal forma que se logró ubicar el centro de masa $(\mathrm{CM})$ a una distancia $e_{s}$ del centro teórico de rigidez. De esta forma fue posible darle al modelo la excentricidad (natural) deseada $e_{s}$ (Figura 4). En este trabajo se usaron dos excentricidades teóricas normalizadas $\left(e=e_{s} / b=0.05\right.$ and 0.15$)$. La rigidez lateral de cada uno de los marcos del modelo fue proporcionada incorporando excentricidades de diseño de acuerdo al Uniform Building Code (ICBO 1997), pero con una excentricidad accidental $e_{a}=0.0$. Ambas excentricidades se pueden expresar de la siguiente forma:

$$
\begin{aligned}
& e_{d 1}=\alpha e_{s}+\beta b=\alpha e_{s}+e_{a} \\
& e_{d 2}=\delta e_{s}+\beta b=\delta e_{s}+e_{a}
\end{aligned}
$$

donde $\alpha$ y $\delta$ son factores de amplificación que modifican la excentricidad estructural estática, mientras que $\beta$ es un factor que toma en cuenta la excentricidad accidental. La cantidad $b$ representa la dimensión en planta del modelo perpendicular a la dirección en que se mide el movimiento ( $b=200 \mathrm{~cm}$, Figura 4).

Para todos los modelos, la excentricidad de diseño $e_{d 1}$ se utilizó para diseñar el marco flexible (placa $\mathrm{F}$ ), mientras que $e_{d 2}$ se utilizó para diseñar el marco rígido (placa R). El uso de dos excentricidades para el diseño de ambos marcos se debe a que la torsión conduce a amplificaciones de fuerzas en el lado flexible y a reducciones de fuerzas en el lado rígido; por lo que el uso de una sola excentricidad no conduciría a las fuerzas de diseño más desfavorables para ambas placas. Las principales características de los modelos se presentan en la Tabla 1. El periodo reportado corresponde a la dirección de la excitación y se calculó mediante una prueba de vibración libre manteniendo fija la base del péndulo, por lo que esta medición corresponde al modelo estructural pero desacoplado del péndulo.

Los valores de $e_{s}$ en esta tabla corresponden a los valores teóricos de la excentricidad natural. De cualquier forma, como se esperaba, subsecuentes pruebas estáticas y dinámicas revelaron que las excentricidades verdaderas resultaron diferentes de estos valores teóricos calculados para fines de diseño. Para los modelos de la Tabla 1, el promedio del valor de la excentricidad accidental (debido a la rigidez solamente) resultó $\left(e_{a}\right)_{a v g}=6.5 \mathrm{~cm}$, el cual correspondió al 3.3\% de la longitud del lado $b$ del modelo. Con base en mediciones de rigidez, en la Tabla 2 se presentan las localizaciones estimadas de los centros de rigidez y de masas de los modelos, donde las distancias reportadas están tomadas respecto al lado izquierdo del modelo (lado rígido en la Figura 4).

Bajo este arreglo de columnas y placas de control, no es difícil observar que las fallas del modelo se concentraron en las placas de control. Por lo tanto, el marco fue prácticamente el mismo para todas las pruebas; sólo se cambiaron las placas de control. Esto hizo posible modificar las propiedades del marco fácilmente, incluyendo la excentricidad $e_{s}$, mediante el ajuste de ambos, la rigidez de las placas de control y la posición de los canales de acero colocados sobre la cubierta (techo) del modelo. 
Tabla 1. Características de los modelos y de sus placas de control.

\begin{tabular}{|c|c|c|c|c|c|c|c|c|}
\hline \multirow[t]{2}{*}{ Modelo } & \multirow[t]{2}{*}{$e_{s}$} & \multicolumn{2}{|c|}{ Lado rígido $(\mathrm{cm})$} & \multicolumn{2}{|c|}{ Lado flexible (cm) } & \multicolumn{2}{|c|}{$\begin{array}{c}\text { Factores para el } \\
\text { diseño por torsión }\end{array}$} & \multirow{2}{*}{$\begin{array}{l}\text { Periodo } \\
\text { medido } \\
\text { (s) }\end{array}$} \\
\hline & & $b_{p l}$ & $t_{p l}$ & $b_{p l}$ & $t_{p l}$ & $\alpha$ & $\delta$ & \\
\hline M1 & $0.05 \times 200=10$ & 3.20 & 1.59 & 5.10 & 1.27 & 1.5 & 1.0 & 0.71 \\
\hline M2 & $0.05 \times 200=10$ & 4.60 & 1.59 & 3.30 & 1.27 & 1.5 & 0.0 & 0.64 \\
\hline M3 & $0.05 \times 200=10$ & 2.30 & 1.90 & 3.00 & 1.59 & 1.0 & 1.0 & 0.64 \\
\hline M4 & $0.05 \times 200=10$ & 3.45 & 1.90 & 1.65 & 1.59 & 1.0 & 0.0 & 0.62 \\
\hline M5 & $0.15 \times 200=30$ & 2.35 & 1.90 & 9.35 & 0.95 & 1.5 & 1.0 & 0.73 \\
\hline M6 & $0.15 \times 200=30$ & 4.40 & 1.59 & 8.70 & 0.95 & 1.5 & 0.0 & 0.67 \\
\hline M7 & $0.15 \times 200=30$ & 2.70 & 1.59 & 10.00 & 0.95 & 1.0 & 1.0 & 0.73 \\
\hline M8 & $0.15 \times 200=30$ & 3.20 & 1.90 & 4.00 & 1.27 & 1.0 & 0.0 & 0.66 \\
\hline
\end{tabular}

Tabla 2. Localización de los centros de rigidez $\left(\mathrm{x}_{\mathrm{CS}}\right)$ y de masas $\left(\mathrm{x}_{\mathrm{CM}}\right)$

\begin{tabular}{cccc}
\hline Modelo & $\begin{array}{c}\text { Centro de } \\
\text { rigidez } \\
\mathrm{x}_{\mathrm{CS}}(\mathrm{cm})\end{array}$ & $\begin{array}{c}\text { Excentricidad } \\
e_{s}(\mathrm{~cm})\end{array}$ & $\begin{array}{c}\text { Centro de masas } \\
\text { (cm) }\end{array}$ \\
\hline M1 & 89.68 & 10.0 & 99.68 \\
M3 & 53.52 & 10.0 & 63.52 \\
M4 & 86.64 & 10.0 & 96.64 \\
M5 & 43.80 & 10.0 & 53.80 \\
\hline M6 & 66.43 & 30.0 & 96.43 \\
M7 & 59.35 & 30.0 & 89.35 \\
M8 & 88.26 & 30.0 & 118.26 \\
\hline
\end{tabular}

\section{RESPUESTA ANALÍTICA}

En esta sección se presenta una solución analítica de la respuesta del sistema (plataforma + modelo), la cual se utiliza para dar soporte a los resultados medidos experimentalmente. El primer paso para obtener la respuesta analítica de la plataforma y del modelo estructural fue construir un modelo analítico, simple, representativo del sistema. En la Figura 7 se pueden observar los grados de libertad que definen este modelo. La variable $X_{1}$ es el desplazamiento lateral total (relativo al piso) de la plataforma. Obviamente, el desplazamiento vertical asociado a la plataforma no se considera como otro grado de libertad, ya que es función tanto del desplazamiento $X_{1}$ como de la longitud de los tirantes de los cuales cuelga la plataforma. En este caso, $L$ representa la longitud del péndulo medida al centro de masa de la plataforma y del generador. El segundo grado de libertad $X_{2}$ es el desplazamiento lateral total de la cubierta o techo del modelo. Aunque el techo del modelo muestra un comportamiento torsional (alrededor de un eje vertical) en este artículo sólo se considera el desplazamiento lateral del centro de masas del modelo. Por lo tanto, el modelo se pudo estudiar con dos grados de libertad. Una descripción de la respuesta torsional se presenta en De-la-Colina et al. (2007). En este caso, $X_{1}$ se define como el desplazamiento lateral de la masa $m_{1}$ asociada a la plataforma y al generador y $X_{2}$ es el desplazamiento lateral total del centro de masas de la cubierta o techo del modelo $\left(m_{2}\right)$.

Las columnas del modelo (con sus placas de control) son los elementos que proveen la rigidez que restaura el desplazamiento lateral de la cubierta del modelo, relativo a la plataforma. En la Figura 7, se 
denota a esta rigidez lateral como $k_{2}$. Por otra parte, la fuerza restauradora de la plataforma está dada por la aceleración de la gravedad $g=9.81 \mathrm{~m} / \mathrm{s}^{2}$, y se puede demostrar fácilmente que una rigidez lateral equivalente (asociada a $X_{1}$ ) se puede estimar mediante la siguiente relación

$$
k_{1}=m_{1}\left(\frac{g}{L}\right)
$$

Esta rigidez se puede obtener sabiendo que la frecuencia angular para el péndulo (solo, sin modelo estructural) es (Timoshenko y Young 1959)

$$
\omega_{P}=\sqrt{\frac{g}{L}}
$$

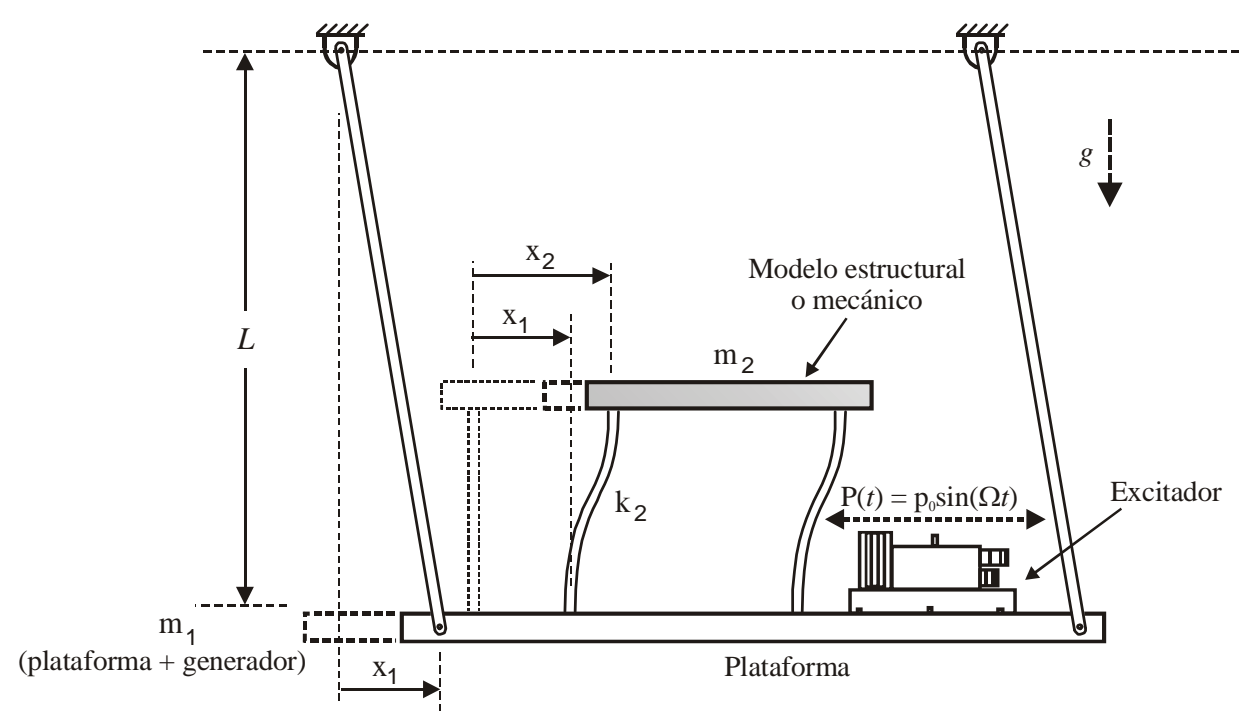

Figura 7. Grados de libertad del sistema.

Con la descripción previa, es posible formular un modelo analítico simple (Figura 8) para representar el comportamiento en el plano del sistema. Nótese que para una apropiada representación de la dinámica del péndulo de prueba, la posición de la segunda masa $m_{2}\left(X_{2}\right)$ puede ser menor que aquella de la primera masa $m_{1}\left(X_{1}\right)$ en el modelo de la Figura 8. Más aún, al inicio del movimiento estas dos coordenadas son iguales a cero. Se han introducido dos amortiguadores viscosos al modelo para tomar en cuenta las fuerzas disipativas. Las constantes de amortiguamiento se denotan como $c_{1}$ y $c_{2}$ las cuales se consideran aproximadamente como $\quad c_{1}=2 \xi_{1}\left(k_{1} / m_{1}\right)^{1 / 2} m_{1}$ y $\quad c_{2}=2 \xi_{2}\left(k_{2} / m_{2}\right)^{1 / 2} m_{2}$ donde $\xi_{1}$ y $\xi_{2}$ son los coeficientes de amortiguamiento expresados como fracción del amortiguamiento. Como se explicó anteriormente, la fuerza lateral $P(t)$ inducida por el generador se define de acuerdo a la Ecuación 1 . De acuerdo al modelo físico, esta fuerza del generador se aplica directamente a la masa $m_{1}$ (plataforma + generador).

Las ecuaciones del movimiento para el péndulo de prueba y para el modelo sencillo de la Figura 8 se formulan de la siguiente forma 


$$
\begin{aligned}
& \ddot{x}_{1} m_{1}+c_{1} \dot{x}_{1}+\left(k_{1}+k_{2}\right) x_{1}-k_{2} x_{2}+p_{0} \operatorname{sen}(\Omega t)=0 \\
& \ddot{x}_{2} m_{2}+c_{2}\left(\dot{x}_{2}-\dot{x}_{1}\right)+k_{2}\left(x_{2}-x_{1}\right)=0
\end{aligned}
$$

Estas ecuaciones se obtuvieron asumiendo comportamiento elástico y pequeños desplazamientos del sistema. Considerando sólo el estado estable de las vibraciones forzadas se puede expresar una solución particular para la Ecuación 6 de la siguiente forma

$$
\begin{aligned}
& x_{1}=A_{1} \cos (\omega t)+A_{2} \operatorname{sen}(\omega t) \\
& x_{2}=A_{3} \cos (\omega t)+A_{4} \operatorname{sen}(\omega t)
\end{aligned}
$$

Sustituyendo estas expresiones en la Ecuación 6 e igualando a cero los coeficientes de $\operatorname{sen}(\omega t)$ y $\cos (\omega t)$, se obtienen cuatro ecuaciones algebraicas lineales que pueden usarse para obtener las cuatro constantes $A_{1}, \ldots, A_{4}$. Resolviendo estas ecuaciones y sustituyendo los valores de $A_{1}, \ldots, A_{4}$ en las Ecuaciones 7 se obtiene la solución del sistema para vibración forzada. En el Apéndice A se presentan las expresiones generales que se obtienen tanto para las constantes $A_{i}$ como para las funciones que definen las coordenadas $X_{1}$ y $X_{2}$. Como se dijo, esta solución analítica del sistema que se presenta en la Figura 8, fue presentada anteriormente por Timoshenko y Young (1959).

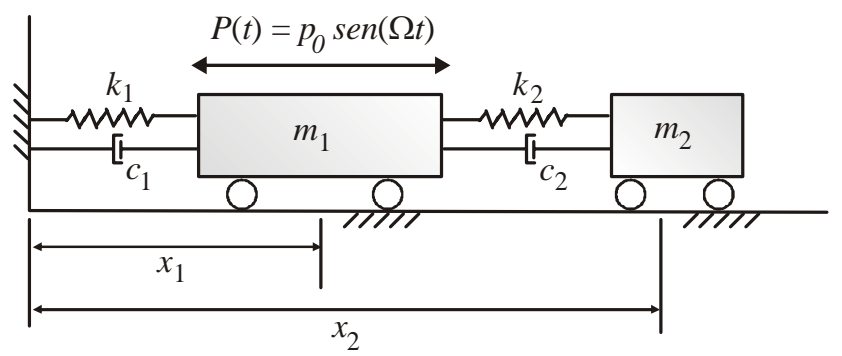

Figura 8. Modelo analítico simple del sistema

La Figura 9 muestra la respuesta analítica basada en la rigidez medida y la frecuencia calculada $\widetilde{\omega}_{6}$ para el modelo M6 con: $m_{1}=991 \mathrm{~N} \cdot \mathrm{s}^{2} / \mathrm{m}\left(1.01 \mathrm{~kg} \cdot \mathrm{s}^{2} / \mathrm{cm}\right), m_{2}=510 \mathrm{~N} \cdot \mathrm{s}^{2} / \mathrm{m}\left(0.52 \mathrm{~kg} \cdot \mathrm{s}^{2} / \mathrm{cm}\right), k_{1}=2,884 \mathrm{~N} / \mathrm{m}$ $(2.94 \mathrm{~kg} / \mathrm{cm}), k_{2}=49,825 \mathrm{~N} / \mathrm{m}(50.79 \mathrm{~kg} / \mathrm{cm}), c_{1}=33.35 \mathrm{~N} \cdot \mathrm{s} / \mathrm{m}(0.034 \mathrm{~kg} \cdot \mathrm{s} / \mathrm{cm}), c_{2}=1,000 \mathrm{~N} \cdot \mathrm{s} / \mathrm{m}(1.02$ $\mathrm{kg} \cdot \mathrm{s} / \mathrm{cm})$ y $\widetilde{\mathrm{p}}_{0}=427 \mathrm{~N}(43.53 \mathrm{~kg})$. Este valor de $\widetilde{\omega}_{6}=9.81 \mathrm{rad} / \mathrm{s}$ es ligeramente diferente al de la frecuencia obtenida con el periodo lateral medido que se reporta en la Tabla 1 ( $\left.\omega_{6}=9.38 \mathrm{rad} / \mathrm{s}\right)$. Estos valores de frecuencia asumiendo para este ejemplo que $\Omega=\omega($ o $\widetilde{\Omega}=\widetilde{\omega})$, llevan a dos fuerzas del generador diferentes: $p_{0}=390 \mathrm{~N}$ y $\widetilde{\mathrm{p}}_{0}=427 \mathrm{~N}$. Por otra parte, la Figura 10 muestra la respuesta registrada para el modelo M6.

Comparando las respuestas de las Figuras 9 y 10, se observa que ambas respuestas (analítica y registrada), son muy parecidas la una a la otra, particularmente para el intervalo entre 5 y $27 \mathrm{~s}$ en el cual la respuesta registrada alcanza el estado estable. Para un tiempo $t<5 \mathrm{~s}$, la respuesta registrada se encuentra en el estado transitorio de vibración, mientras que para $t>27 \mathrm{~s}$ la fuerza del excitador ha 
disminuido (casi vibración libre). Esto explica porqué la solución analítica no puede reproducir exactamente la respuesta completa registrada.

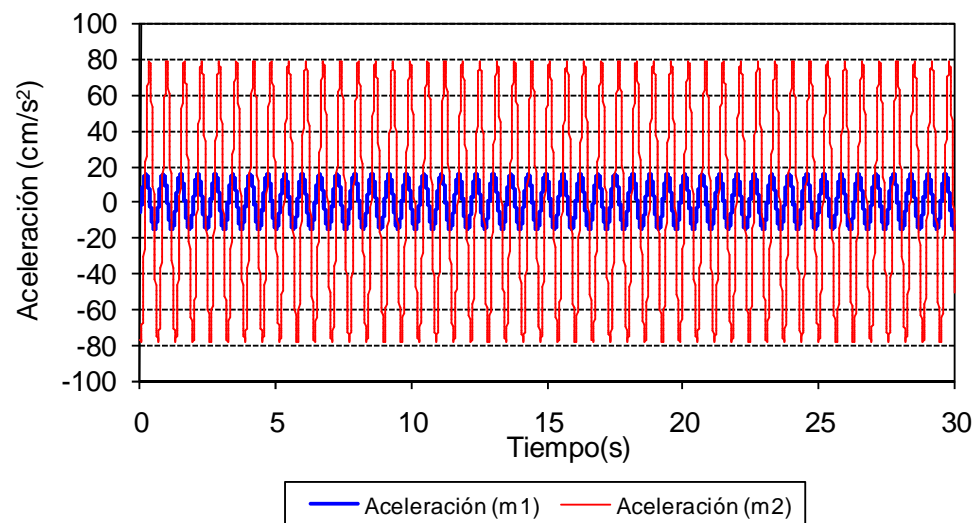

Figura 9. Respuesta analítica del péndulo de prueba.

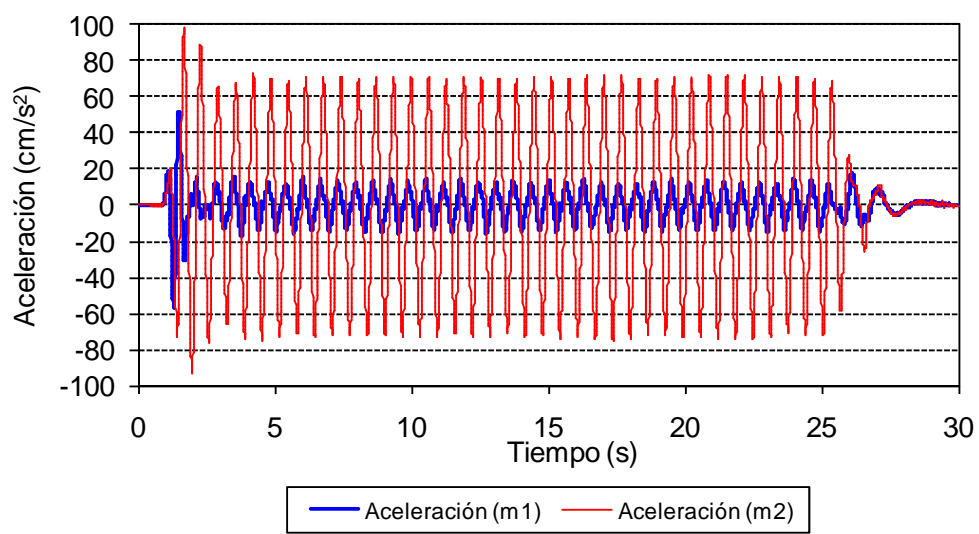

Figura 10. Respuesta registrada durante una prueba específica en el péndulo de prueba.

El modelo que se probó estuvo instrumentado solamente con dos acelerómetros localizados sobre los marcos laterales (ver acelerómetros 1 y 2 en la Figura 4b). La aceleración de la masa $m_{2}$, la cual representa la aceleración en el centro de masas del modelo, se estimó mediante una interpolación de los registros de aceleración 1 y 2.

En la Figura 11 se muestran dos gráficas donde se compara la aceleración experimental con la analítica. La gráfica de la izquierda corresponde a la masa $m_{1}$ y la de la derecha a la masa $m_{2}$. Al igual que en la gráficas mostradas anteriormente, se aprecia que con excepción de la fase transitoria de la respuesta, ambas señales (analítica y experimental) son similares entre sí, lo cual revela la precisión en la respuesta estacionaria del modelo analítico desarrollado. 

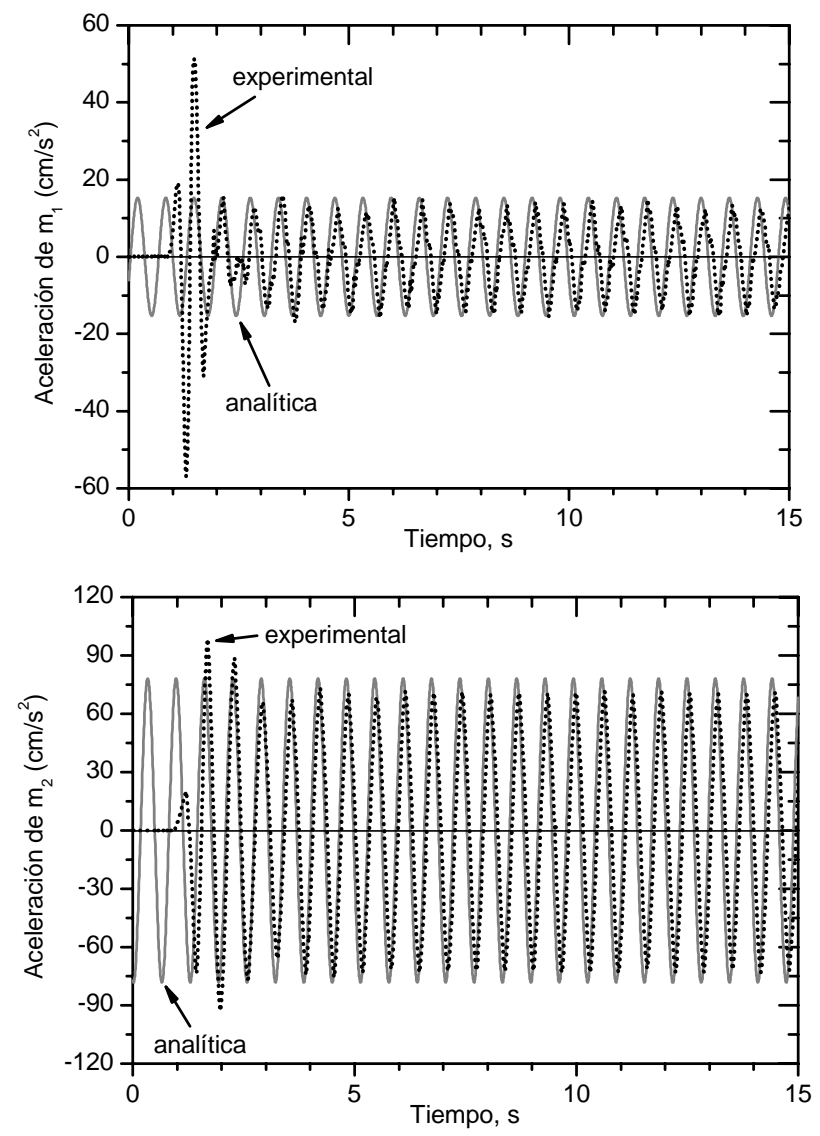

Figura 11. Comparación de aceleraciones (experimental y analítica).

\section{RESULTADOS}

En esta sección, se describen en dos partes los resultados de las pruebas dinámicas llevadas a cabo. Primero se describe y analiza el movimiento de la plataforma para caracterizar el tipo de movimiento (del suelo) que simula el péndulo de prueba. En esta parte se analizan los espectros de respuesta y de Fourier calculados a partir de las aceleraciones registradas en la plataforma del péndulo, ello con el propósito de compararlos con los espectros calculados para distintos tipos de terreno durante la ocurrencia de sismos reales. En la segunda parte, se presenta una descripción global de la respuesta de los modelos con el propósito de ilustrar sus características. En este trabajo no se presentan con detalle los resultados de torsión de los modelos estructurales probados; para mayor información al respecto se puede consultar el trabajo de De la Colina et al. (2007).

\section{Movimiento de la plataforma}

El propósito de este análisis es identificar el tipo de movimiento que el péndulo de pruebas experimentales reproduce en su plataforma. Para caracterizar el movimiento del suelo, en este caso el generado en la plataforma, es conveniente el uso de espectros de respuesta y de Fourier. 
Cuando la excitación es causada por el movimiento del suelo durante un temblor, la forma del espectro de respuesta se afecta significativamente por las características del suelo. Un típico ejemplo es el espectro de respuesta en terreno blando de la ciudad de México durante el temblor de 1985 ocurrido en México $(M=8.1)$. Mientras que en terreno duro se observaron aceleraciones del suelo cercanas a $0.05 \mathrm{~g}$, en suelo blando se registraron aceleraciones hasta cinco veces mayores, además de que en suelo blando el movimiento del suelo resultó similar a una onda de tipo armónico. La forma del espectro de respuesta en este caso resultó con máximas respuestas para periodos entre 2 y $3 \mathrm{~s}$. Por lo que se refiere a las ordenadas espectrales (seudoaceleraciones) la amplificación en terreno blando llegó a ser de hasta más de ocho veces respecto a terreno duro. La influencia de las condiciones del suelo en la forma del espectro de respuesta se ilustra en la Figura 12, la cual muestra los espectros de respuesta (seudoaceleraciones) de osciladores de un grado de libertad con $\xi=5 \%$ (fracción del amortiguamiento crítico) para sitios de suelo blando (estación SCT) y duro (estación CU) correspondientes al temblor del 19 de septiembre de 1985, así como el espectro de respuesta calculado a partir de las aceleraciones registradas en la plataforma del péndulo durante una de las pruebas. En este caso el eje vertical muestra la relación de la seudoaceleración de la masa del oscilador $(S a)$ respecto a la máxima seudoaceleración $\left(S a_{m}\right)$ de todo el espectro, mientras que el eje horizontal corresponde al periodo de vibración del oscilador $(T)$ normalizado respecto al periodo para el cual se alcanzó la máxima seudoaceleración $\left(T_{m}\right)$. Es claro que en el caso de la plataforma, el periodo $T_{m}$ es aquel correspondiente a la frecuencia de operación del excitador mecánico. La Figura 12 muestra que el espectro SCT concentra los máximos valores en una banda estrecha de periodos, mientras que el espectro de CU no lo hace. Se considera que ésta es una de las principales características que distingue a los espectros de respuesta de terreno blando respecto a los de terreno duro. Por otra parte, en la misma Figura 12a se aprecia que la forma del espectro que se obtiene a partir de las aceleraciones registradas en la plataforma del péndulo es muy parecido al de SCT (terreno blando), en el sentido de que el espectro crece rápidamente del valor correspondiente a $T / T_{m}=0.0$ hasta el valor máximo en $T / T_{m}=1.0$ y posteriormente disminuye en forma asintótica hacia valores de 0.0 para valores crecientes de $T / T_{m}$. Es claro que existen diferencias menores entre ambos espectros ya que uno pertenece a un movimiento quasi-armónico y el otro a un sismo.

La Figura 13 muestra la aceleración del suelo $\left(A_{s}\right)$ para las mismas dos estaciones (SCT y CU) durante el mismo temblor. Como se esperaba de los espectros de respuesta mostrados anteriormente, el registro de la estación SCT (terreno blando) se aproxima más a una función armónica de amplitud variable en comparación al registro de CU. Esto se aprecia ya que el registro de SCT tiene picos de aceleración casi igualmente espaciados (periódicos), mientras que el de CU no. Más aún, se puede identificar para el registro de SCT un periodo cercano a los $2 \mathrm{~s}$. De acuerdo a esta comparación entre espectros de respuesta y registros de aceleraciones, se observa que el movimiento de la plataforma del péndulo se asemeja más al de terreno blando, que al de terreno duro.

La Figura 14 permite comparar el espectro de amplitudes de Fourier del movimiento típico de la plataforma del péndulo de prueba con el obtenido en la estación SCT durante el temblor de 1985. Ambos espectros muestran un intervalo de banda angosta en el cual el contenido de energía es alto en comparación al observado para otras frecuencias. La forma característica de ambos espectros de amplitudes de Fourier ratifica la similitud observada entre el movimiento de la plataforma del péndulo respecto al que se presenta en terreno blando durante la ocurrencia de sismos reales. Por otro lado, se aprecia una diferencia importante en los acelerogramas que se muestran en la misma Figura 14; mientras la fase intensa del movimiento para el caso de la plataforma se presenta al inicio de la señal, en el caso del sitio SCT la fase intensa se presenta en la parte intermedia del registro. Esta característica puede ser relevante por lo que deberá tomarse en cuenta en las pruebas que se realicen con el péndulo. 


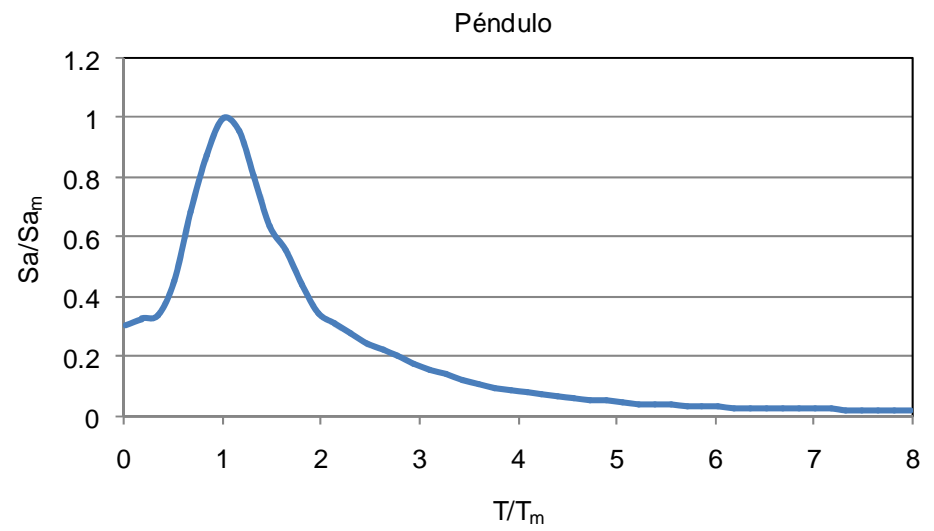

a) Plataforma del péndulo de prueba $\left(\mathrm{T}_{\mathrm{m}}=0.37 \mathrm{~s}\right)$.

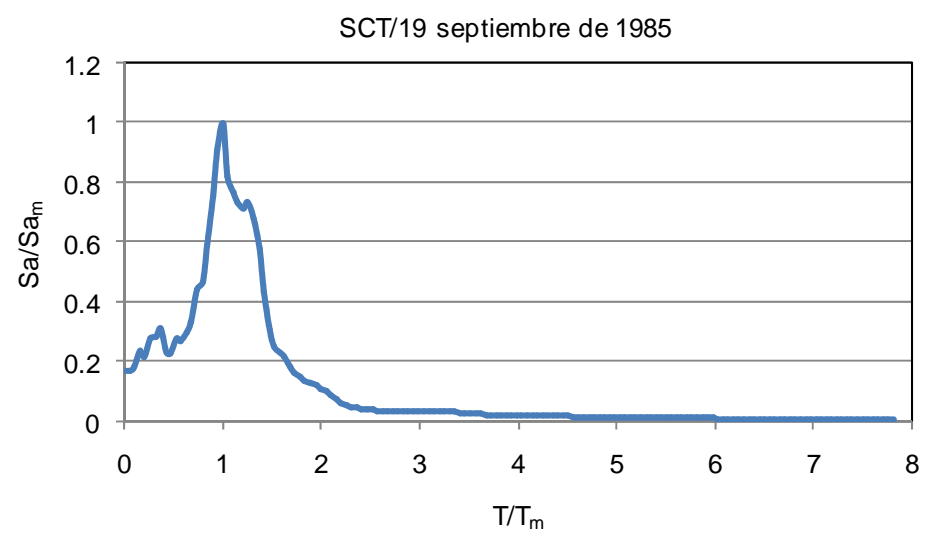

b) Sitio SCT (terreno blando, $\mathrm{T}_{\mathrm{m}}=2.0 \mathrm{~s}$ ).

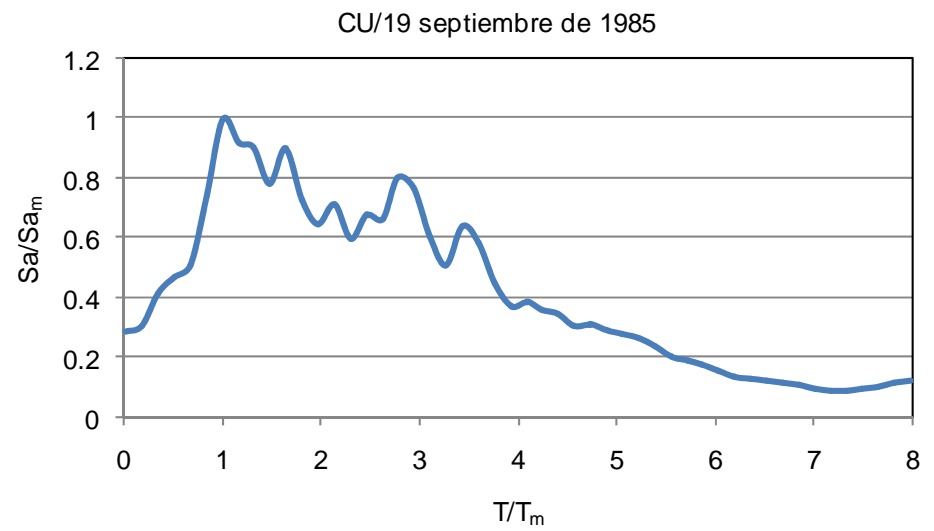

c) Sitio CU (terreno duro, $\mathrm{T}_{\mathrm{m}}=0.37 \mathrm{~s}$ ).

Figura 12. Espectros de respuesta normalizados calculados a partir de las aceleraciones registradas en dos sitios durante el temblor de 1985 en México, así como para las aceleraciones registradas en la plataforma del péndulo durante una de las pruebas. 

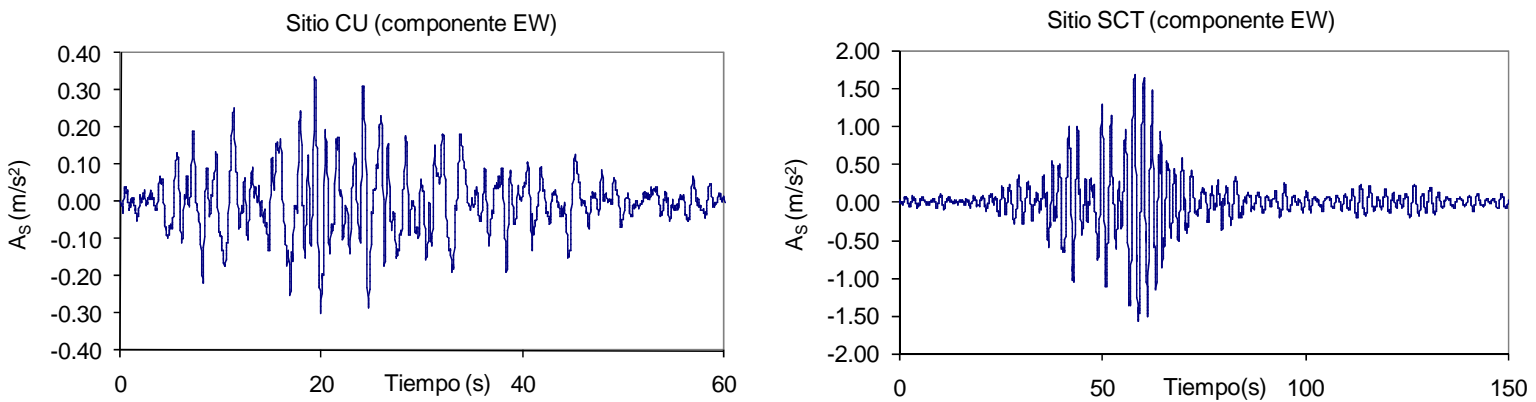

Figura 13. Registros de aceleraciones en dos sitios distintos de la ciudad de México durante el temblor de 1985.
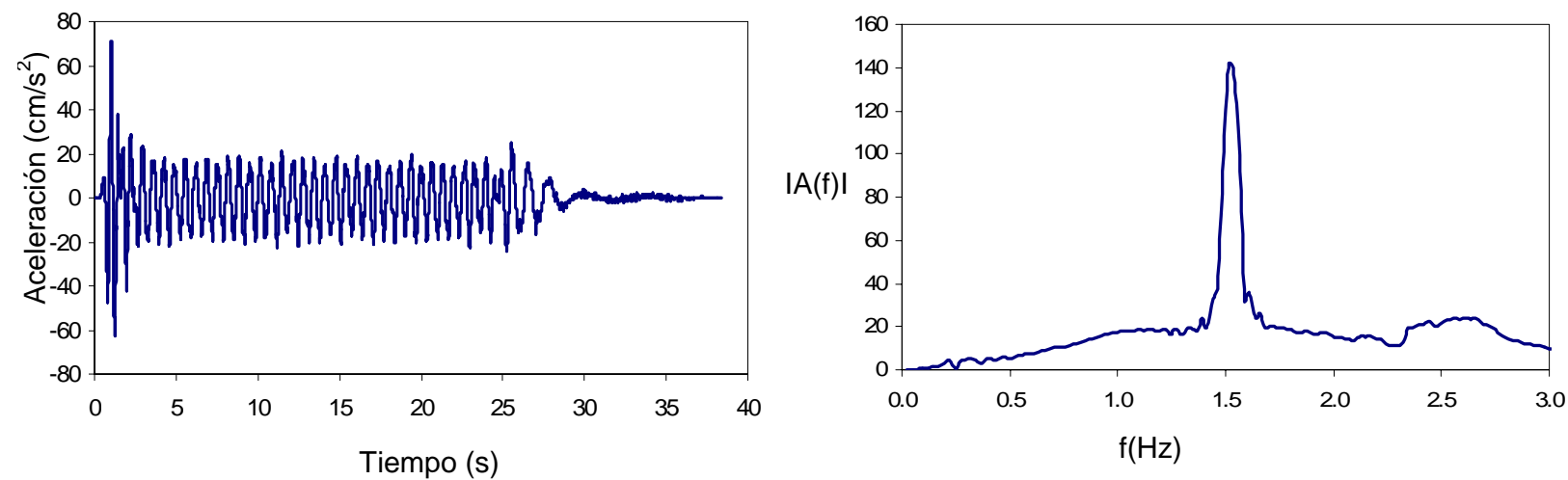

a) Resultados típicos de la plataforma.
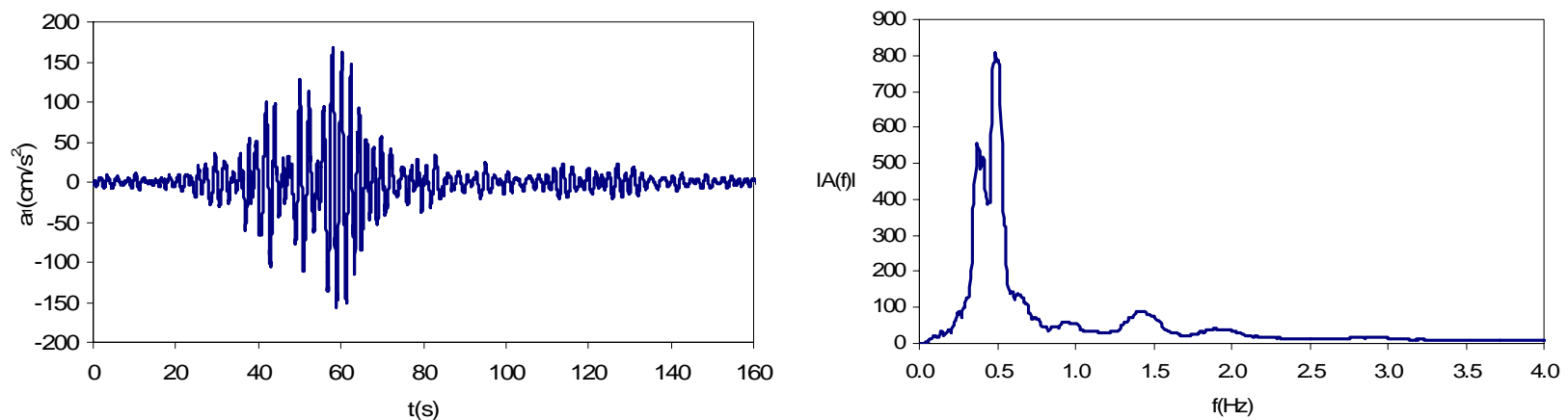

b) Registro del temblor de 1985 en México para terreno blando de la ciudad de México (SCT, $\mathrm{T}_{\mathrm{s}}=2.0 \mathrm{~s}$ ).

Figura 14. Registros de aceleraciones y espectros de amplitudes de Fourier (plataforma del péndulo y estación SCT). 


\section{Respuesta de los modelos}

En esta sección se describen algunos de los resultados de la respuesta de los modelos ensayados. Como se señaló al inicio de esta sección, el objetivo de presentar los resultados de los modelos es hacer notar que los resultados muestran características propias de estructuras desplantadas en terreno blando.

La Figura 15 muestra los registros de la respuesta (aceleraciones), tanto de un oscilador de un grado de libertad ( $\mathrm{T}=2.0 \mathrm{~s}$ ) sometido al movimiento del suelo en la estación SCT de la ciudad de México durante el temblor de 1985 (suelo blando), como el de uno de los modelos ensayados en el péndulo de prueba $(\Omega=\omega=9.38 \mathrm{rad} / \mathrm{s})$. Se observa que a pesar de que las amplitudes de la respuesta del modelo M6 se mantienen constantes durante casi la totalidad de la prueba, lo cual no sucede en el oscilador sujeto al registro SCT, en el dominio de la frecuencia se observa que ambas señales resultan de banda angosta, apreciándose que las máximas amplitudes del espectro de la señal corresponden con los periodos de vibración, tanto del oscilador (Fig. 15a) como del modelo M6 (Fig. 15b). Esto sugiere que el péndulo de prueba induce respuestas estructurales similares en frecuencia a las que se observan en estructuras desplantadas en terreno blando.
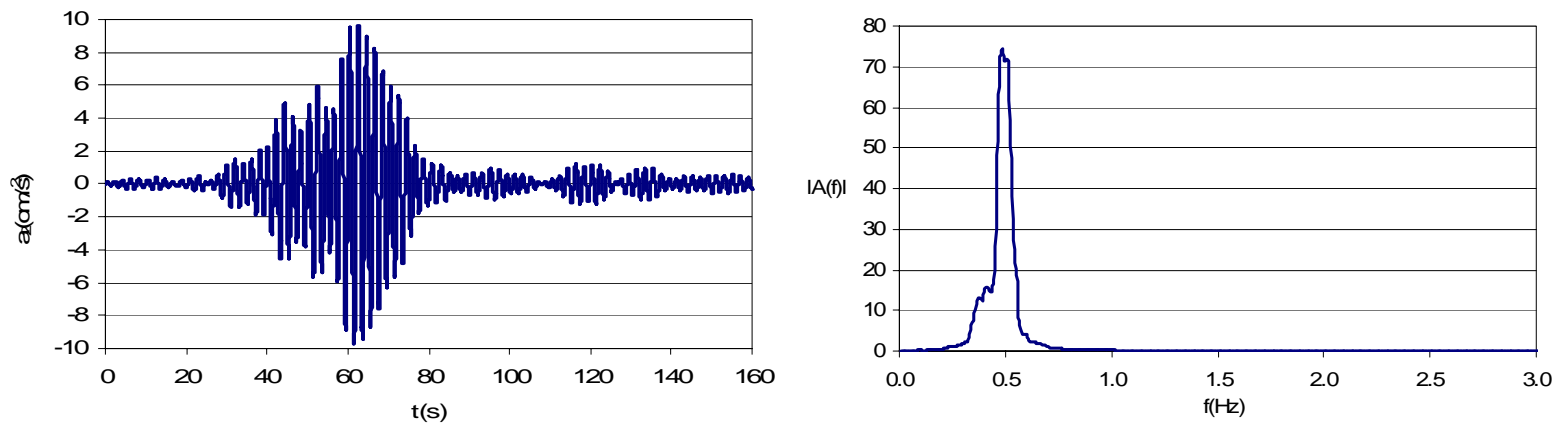

a) Respuesta de un oscilador de un grado de libertad ( $\mathrm{T}=2.0 \mathrm{~s}$ ) sometido al movimiento del suelo en la estación SCT de la ciudad de México durante el temblor de 1985 (suelo blando).
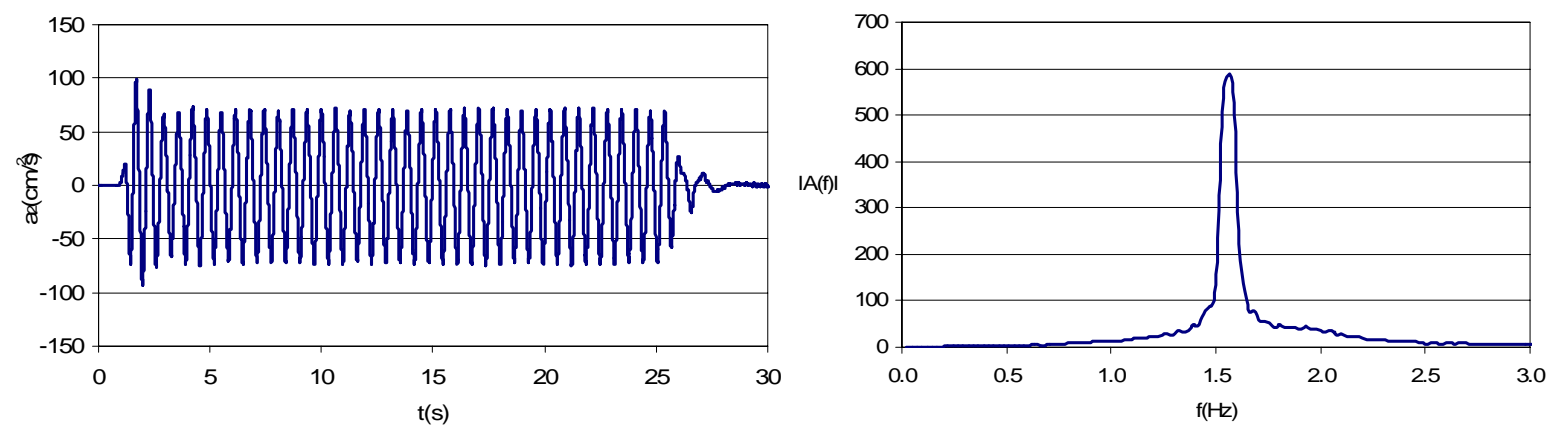

b) Respuesta del modelo M6 ensayado en el péndulo de prueba ( $\Omega=\omega=9.38 \mathrm{rad} / \mathrm{s})$.

Figura 15. Registros de aceleraciones y espectros de amplitudes de Fourier. 
La Figura 16 muestra algunos espectros de respuesta normalizados respecto a la máxima aceleración del suelo para temblores registrados en diferentes sitios. Esta figura también muestra los valores normalizados de las máximas aceleraciones del centro de masa $\left(m_{2}\right)$ de los modelos ensayados respecto a la máxima aceleración de la plataforma. Los cuadros negros corresponden a los cocientes calculados con los valores máximos registrados durante la respuesta total (transitoria y estacionaria) del modelo. A su vez, los cuadros blancos corresponden a los cocientes calculados tomando en cuenta solamente la parte estacionaria de la vibración del modelo. Excepto por el modelo M4 ( $T=0.62 \mathrm{~s})$ los resultados de los modelos corresponden a tres distintas relaciones de frecuencia $(\Omega / \omega=0.8,1.0$ y 1.2). Para el modelo M4 sólo se muestran resultados para $\Omega / \omega=1.0$ y 1.2, por lo que sólo aparecen 2 cuadros blancos y dos cuadros negros en la Fig. 16 para este periodo. La información de los registros sísmicos se muestra en la Tabla 3.

Tabla 3. Información de los registros sísmicos de la Fig. 16

\begin{tabular}{|c|c|c|}
\hline Estación & Sismo & $\begin{array}{l}\text { Magnitud } \\
\left(\mathrm{M}_{\mathrm{s}}\right)\end{array}$ \\
\hline Presidio & $\begin{array}{c}\text { Loma Prieta, } \\
\text { California }(17 / 10 / 1989)\end{array}$ & 7.0 \\
\hline El Centro & $\begin{array}{c}\text { Imperial Valley, } \\
\text { California (18/05/1940) }\end{array}$ & 7.1 \\
\hline $\begin{array}{l}\text { Caleta, CU, } \\
\text { SCT y Central de } \\
\text { Abastos }\end{array}$ & $\begin{array}{l}\text { Michoacán, México } \\
\text { (19/09/1985) }\end{array}$ & 8.1 \\
\hline
\end{tabular}

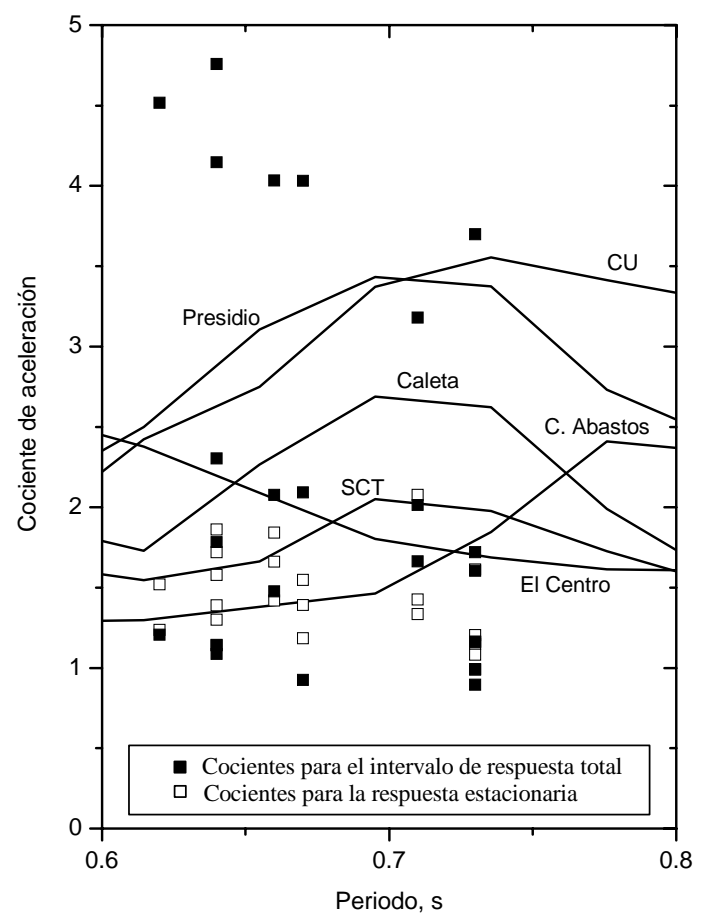

Figura 16. Relaciones de aceleraciones (centro de masa /base) y espectro de respuesta para distintos registros sísmicos. 
Se puede observar que para este intervalo de periodos de vibración ( 0.6 a 0.8 s), el cual incluye los periodos de todos los modelos ensayados, los cocientes para el estado estacionario (cuadros blancos) se encuentran cercanos a los cocientes obtenidos de los registros sísmicos de terreno blando (estaciones SCT y Central de Abastos). Esto permite sugerir que el tipo de movimiento que el péndulo de prueba impone a los modelos, considerando sólo la fase estacionaria, es similar a los registrados en suelos blandos durante la ocurrencia de temblores.

\section{CONCLUSIONES}

En este artículo, se presentó y analizó un aparato sencillo y de bajo costo para el estudio dinámico en laboratorio de modelos estructurales y mecánicos. Este sencillo péndulo de prueba se utilizó para llevar a cabo pruebas experimentales de sistemas torsionalmente desbalanceados de un nivel. Las respuestas del modelo y de la plataforma del péndulo de prueba se midieron y compararon respecto a la solución analítica de un modelo equivalente de dos grados de libertad.

Los resultados obtenidos tanto de los modelos como del sistema muestran el potencial que este equipo de pruebas puede tener para fines de investigación o educación en dinámica estructural e ingeniería sísmica.

Las principales conclusiones de este trabajo son las siguientes:

1. La forma del espectro de respuesta de un oscilador que se obtiene a partir de las aceleraciones registradas en la plataforma del péndulo es muy parecido a la forma del espectro calculado a partir de las aceleraciones registradas en la estación SCT (terreno blando) durante el sismo de 1985; en el sentido de que el espectro crece rápidamente del valor correspondiente a $T / T_{m}=0.0$ hasta el valor máximo en $T / T_{m}=1.0$ y posteriormente disminuye en forma asintótica hacia valores de 0.0 para valores crecientes de $T / T_{m}$ ( $T$ es el periodo del oscilador y $T_{m}$ el periodo del oscilador para el cual se alcanzó la máxima seudoaceleración).

2. De acuerdo a la comparación entre espectros de respuesta y registros de aceleraciones, se observa que el movimiento de la plataforma del péndulo se asemeja más al de terreno blando, que al de terreno duro. La forma característica de los espectros de amplitudes de Fourier ratifica la similitud observada entre el movimiento de la plataforma del péndulo respecto al que se presenta en terreno blando durante la ocurrencia de sismos reales.

3. Por lo que respecta a la forma de los acelerogramas de los registros sísmicos en terreno blando y aquellos obtenidos en la plataforma del péndulo, se observa que la fase intensa del movimiento de la plataforma se presenta al inicio de la señal, mientras que para los registros de terreno blando, la fase intensa se presenta en la parte intermedia del registro.

4. La comparación del espectro de Fourier de la respuesta de un oscilador de un grado de libertad sometido a movimiento sísmico en terreno blando respecto al espectro de Fourier de la respuesta de los modelos ensayados en el péndulo de pruebas, indica que el péndulo de prueba induce respuestas estructurales similares en frecuencia a las que se observan en estructuras desplantadas en terreno blando.

5. Con base en la comparación de las respuestas máximas normalizadas de los modelos ensayados en el péndulo de pruebas, respecto a las respuestas calculadas a partir de registros sísmicos de terreno 
blando, se observa que el tipo de movimiento que el péndulo de prueba impone a los modelos considerando sólo la fase estacionaria, conduce a amplificaciones de la respuesta del mismo orden a las obtenidas en el caso de movimientos en terreno blando.

6. Se observó que el dispositivo de prueba no es apropiado para reproducir movimientos registrados en terreno firme.

\section{APÉNDICE A}

$$
\begin{gathered}
x_{1}=A_{1} \cos (\omega t)+A_{2} \sin (\omega t) \quad x_{2}=A_{3} \cos (\omega t)+A_{4} \sin (\omega t) \\
\dot{x}_{1}=-A_{1} \omega \sin (\omega t)+A_{2} \omega \cos (\omega t) \\
\dot{x}_{2}=-A_{3} \omega \sin (\omega t)+A_{4} \omega \cos (\omega t) \quad x_{1}=-A_{1} \omega^{2} \cos (\omega t)-A_{2} \omega^{2} \sin (\omega t) \\
x_{2}=-A_{3} \omega^{2} \cos (\omega t)-A_{4} \omega^{2} \sin (\omega t)
\end{gathered}
$$

donde:

$$
\begin{aligned}
& A_{1}=\frac{P\left(-k_{1} k_{2}^{2}+m_{2}^{2}\left(\omega^{6} m_{1}-\omega^{4} k_{1}-\omega^{4} k_{2}\right)+m_{2} k_{2}\left(-2 \omega^{4} m_{1}+2 \omega^{2} k_{1}+\omega^{2} k_{2}\right)+m_{1} \omega^{2} k_{2}^{2}+c_{2}^{2} \omega^{4} m_{1}-c_{2}^{2} \omega^{2} k_{1}\right)}{B} \\
& A_{2}=-\frac{\left(c_{1} m_{2}^{2} \omega^{4}+\omega^{2} c_{1} c_{2}^{2}+m_{2} \omega^{2} k_{2} c_{2}-2 \omega^{2} m_{2} k_{2} c_{1}+c_{1} k_{2}^{2}\right) \omega P}{B} \\
& A_{3}=\frac{P\left(-m_{2} \omega^{4} m_{1} k_{2}+m_{2} \omega^{2} k_{1} k_{2}+m_{2} \omega^{4} c_{2} c_{1}+m_{2} \omega^{2} k_{2}^{2}+m_{1} \omega^{2} k_{2}^{2}-k_{1} k_{2}^{2}+c_{2}^{2} \omega^{4} m_{1}-c_{2}^{2} \omega^{2} k_{1}\right)}{B} \\
& A_{4}=\frac{\omega P\left(m_{1} \omega^{4} m_{2} c_{2}-c_{1} k_{2}^{2}+\omega^{2} m_{2} k_{2} c_{1}-k_{1} m_{2} \omega^{2} c_{2}-m_{2} \omega^{2} k_{2} c_{2}-\omega^{2} c_{1} c_{2}^{2}\right)}{B} \\
& B=k_{1}^{2} k_{2}^{2}+m_{2}^{2} \omega^{8} m_{1}^{2}-2 m_{2}^{2} \omega^{6} m_{1}\left(k_{1}+k_{2}\right)-2 m_{2} \omega^{6} m_{1}^{2} k_{2}+4 m_{2} \omega^{4} m_{1} k_{2} k_{1}+m_{2}^{2} \omega^{4} k_{1}^{2}+2 m_{2}^{2} \omega^{4} k_{1} k_{2} \\
& -2 m_{2} \omega^{2} k_{1}^{2} k_{2}+m_{2}^{2} \omega^{4} k_{2}^{2}++2 m_{2} \omega^{4} k_{2}^{2} m_{1}-2 m_{2} \omega^{2} k_{2}^{2} k_{1}+2 m_{2} \omega^{4} k_{2} c_{2} c_{1}+m_{1}^{2} \omega^{4} k_{2}^{2}-2 m_{1} \omega^{2} k_{2}^{2} k_{1} \\
& +c_{2}^{2} \omega^{4} c_{1}^{2}+c_{2}^{2} \omega^{6} m_{1}^{2}-2 c_{2}^{2} \omega^{4} m_{1} k_{1}+c_{2}^{2} \omega^{2} k_{1}^{2}+m_{2}^{2} \omega^{6} c_{1}^{2}-2 m_{2} \omega^{4} c_{1}^{2} k_{2}+c_{1}^{2} \omega^{2} k_{2}^{2}
\end{aligned}
$$

\section{REFERENCIAS}

Acuña, Q., 2003, Diseño, construcción y prueba de un excitador mecánico de masas excéntricas, Tesis de Maestría. Universidad Autónoma del Estado de México.

Clough, R.W. y J. Penzien, 1993, Dynamics of Structures, 2nd ed. McGraw-Hill, New York, USA. 
De-la-Colina, J., Q. Acuña, A. Hernández y J. Valdés, 2007, "Laboratory tests of steel simple torsionally unbalanced models,” Earthquake Engineering and Structural Dynamics, Vol. 36-7, pp. 887-907.

Hudson, D. E., 1970, Dynamic tests of full-scale structures, Capítulo 7 in Wiegel, L. W. (editor), Earthquake Engineering, Prentice Hall, Englewood Cliffs, N. J., USA.

Humar, J. y P. Kumar, 1999, "Effect of orthogonal inplane structural elements on inelastic torsional response,” Earthquake Engineering and Structural Dynamics, Vol. 28, pp. 1071-1097.

International Conference of Building Officials (ICBO), 1997, Uniform Building Code. Whittier, California, USA.

Mahin, S. A. y B. P. Shing, B. P, 1985, "Pseudo dynamic method for seismic testing," J. Structural Engineering, ASCE, 111(7), pp. 1482-1503.

Nieto, J. A., J. Cervantes, y J. A. Díaz, 1970, “Excitador mecánico de velocidad controlada para estudios de vibración forzada”, Reporte de Investigación 244, Instituto de Ingeniería, Universidad Nacional Autónoma de México, México.

Newmark, N. M. y W. J. Hall, 1982, Earthquake spectra and design, Monograph, Earthquake Engineering Research Institute, California, USA.

Takanashi, K., K. Udagawa y H. Tanaka, 1980, "Pseudo dynamic tests on a two-story steel frame by a computer-load apparatus hybrid system," Procedings of the. 7th World Conference on Earthquake Engineering, Istambul, Turkey, Vol. 7, pp. 225-232.

Timoshenko, S. y D. H. Young, 1959. Vibration problems in engineering, 3rd. ed., Van Nostrand, New Jersey, USA.

Yu, E., D. Skolnik, D. H. Whang y J. W. Wallace, 2008, "Forced vibration testing of a four-story reinforced concrete building utilizing the nees@UCLA mobile field laboratory.” Spectra, Vol. 24-4, pp.969-996. 